\title{
Dysregulation of miR144 and miR451 Expression in the Circulating Human Erythrocytes from the African American Adults
}

\author{
Ghadi Alsharif $^{1}$, Ibrahim Jafri ${ }^{1}$, Ronald Berasa ${ }^{2}$, Nesreen Hag Ahmed ${ }^{3}$, Gail Nunlee-Bland ${ }^{3}$, \\ Maurice B. Fluitt ${ }^{4}$, Kanwal K Gambhir ${ }^{* *}$ \\ ${ }^{1}$ Department of Genetics and Human Genetics, Howard University College of Medicine, United States \\ ${ }^{2}$ Department of $\mathrm{OB} / G Y N$, Howard University Hospital, United States \\ ${ }^{3}$ Diabetes Treatment Center, Howard University Hospital, United States \\ ${ }^{4}$ Division Endocrinology and Metabolism, Department of Medicine, Howard University College of Medicine, \\ United States
}

*Corresponding Author: Kanwal K Gambhir, Department of Genetics and Human Genetics, Howard University College of Medicine, United States. Email: kgambhir@howard.edu

\begin{abstract}
There are several mechanisms that drive the aging process in the human body. Cellular senescence which leads to permanent cell growth arrest and oxidative stress are examples of aging promoting mechanisms. Moreover, genetics and epigenetic play important roles in accelerating and/or delaying the onset of the aging process. miRNAs are important players in controlling OS, aging, and cellular senescence. The purpose of this study was to evaluate circulating erythrocyte miR-451 and miR-144 expression as potential biomarker of cell aging. Blood samples were collected from consented volunteers and miRNAs were isolated from a control group and Type 2 Diabetes Mellitus. Taqman primers were used for detection and quantification of miR-144 and miR-451. Erythrocytes were further sub-fractionated into young, mid and old age by using discontinuous Percoll gradient. miRNA's were isolated from erythrocytes using miRNA Isolation kit. The miR-451 expression was significantly down-regulated in old cells in T2DM group, while miR-144 expression was significantly up-regulated in old cells in T2DM. The findings of this study are consistent with our previous report of increase expression of miR-451 and miR-144 as the cell gets old. The current findings suggest that miR-451 and miR-144 may have a role in aging process.
\end{abstract}

Keywords: MicroRNA Expression, Cell Aging, Oxidative stress, microRNA, Erythrocytes, Type 2 Diabetes, Biomarkers of erythrocyte aging.

Abbreviations: Oxidative stress: OS, miRNA: miR, Erythrocytes: E, Young: y, mid: m, Old: o, Type 2 Diabetes Mellitus: T2DM, Red Blood Cells: RBCs, Insulin Receptor Substrate 1: IRS-1, Impaired Fasting, Glucose: IFG, Diabetes Mellitus: DM, Type 1 Diabetes mellitus: T1DM

\section{INTRODUCTION}

Diabetes mellitus (DM) is considered a global public health issue, affecting 463 million adults worldwide [1]. This count is estimated to rise above 640 million people by 2040 [2]. DM is caused by the failure of pancreas cells to produce insulin (Type1) or decrease insulin sensitivity (Type2). DM imposes a series of complex multi-organ and multi-system effects on the body over time and leads to cardiovascular disease, kidney failure, blindness, and nerve damage. Although many clinical procedures have been established to manage and treat DM, the underlying mechanisms of DM and its complications need to be fully understood in order to develop effective therapeutic strategies and identify novel biomarkers of disease [3].

Red blood cells (RBCs) (Erythrocytes) are the most abundant cells in blood stream, accounting for more than $90 \%$ of the cell population in peripheral blood. The function of RBCs is to carry oxygen throughout the body by binding to hemoglobin. Autoxidation of hemoglobin makes RBCs more susceptible to oxidative stress (OS) damage [4]. Recent reports highlight the emerging role of RBCs in several biological processes of importance to metabolic functions, including diabetes mellitus and its complications $[5,6]$. These studies suggest that disturbances in RBC function and structure can promote oxidative stress and jeopardize antioxidant 
protection, resulting in red blood cell dysfunction and injury to peripheral tissues $[5,7]$. Thus, RBCs provide an efficient and attractive means to identify markers and mechanisms of cell aging in the setting of type 2 diabetes mellitus (T2DM). MicroRNAs (miRNA) are a class of small (18-25 nucleotides) noncoding RNA that are important in the regulation of gene expression by inhibiting translation or degrading target mRNAs [8]. MiRNAs are found and function throughout the body in both tissue and circulation. Previous work from our group and others has identified several miRNAs deregulated in T2DM [9-11]. We previously reported reduced expression of several miRNAs in erythrocytes of pre-diabetic adults [9-10], which suggests a potential regulatory role for miRNAs in these cells. MiR-451 and miR-144 are highly expressed and are important miRNAs in RBCs maturation and function [12]. MiR-451 reportedly protects erythrocytes against oxidant stress through a miR-451/14-3-3 $/$ /FoxO3 regulatory axis [12].

Recent evidence suggests its role in the development of T2DM and its micro vascular complications [13-14]. In addition to miR-451, miR-144 has been shown to play an important role in T2DM. Investigators found that high expression of miR-144 significantly inhibit IRS1 mRNA in T2DM and increase the glycemic status in those individuals [15].Moreover, this study identified additional miRs that may participate in the regulation of insulin signaling and serve as biomarkers of disease [15]. The diagnostic, prognostic, and therapeutic potential of miRNAs in both tissue and circulation has been established and documented. However, additional studies are warranted to understand the complex nature and function of miRNAs in T2DM, especially those found in circulation (i.e. micro vesicles and RBCs). These circulating miRNAs can influence several biologically important processes, including cell aging. Moreover, circulating miRNAs provide an attractive early marker of disease progression. In this study, we evaluate miR-451 and miR-144 expression as a potential biomarker of cell aging in erythrocyte sub-fractions (young-, middle-, old-cells) isolated from type 2 diabetic adults.

\section{Materials And Methods}

\subsection{Participants and RBC Samples Processing}

A total of 16 blood samples (T2DM=8, control=8) were collected and a written informed consent was obtained from all. Blood samples $(\sim 20 \mathrm{~mL})$ were collected following an overnight fast of 8-10 hours in tubes containing heparin using a standard procedure. The study protocol was approved by Howard University Institutional Review Board (HUIRB) (IRB-13MED-73). Whole blood was centrifuged for 20minutes at $1200 \mathrm{rpm}$.

The plasma layer was removed and stored at -80 ${ }^{\circ} \mathrm{C}$ for future study. Isotonic choline chloride solution was added to RBCs and mixed gently by inversion. To obtain purified RBCs, RBCs were added to the wall of a glass tube contain a mixture of $1 \mathrm{~mL}$ Hypaque $(\mathrm{H})(33.9 \%)$ and 2.4 $\mathrm{mL}$ Ficoll (F) (9\%) and spun for 20 minutes at $1200 \mathrm{rpm}$. This procedure repeated twice. The purified RBCs separated into three subtractions (1.07-1.09 as y, 1.09-1.11 as $\mathrm{m}$, and 1.11-1.12 as o) by layering them over a percoll gradient consisting of $35 \%, 40 \%, 45 \%, 50 \%, 55 \%, 65 \%$, $80 \%$, and $100 \%$ of percoll and the balance Gibco minimum essential medium [14] as shown in Figure 1. This was centrifuged at 1200 rpm for 30 minutes. Then the respective age subtractions were collected.

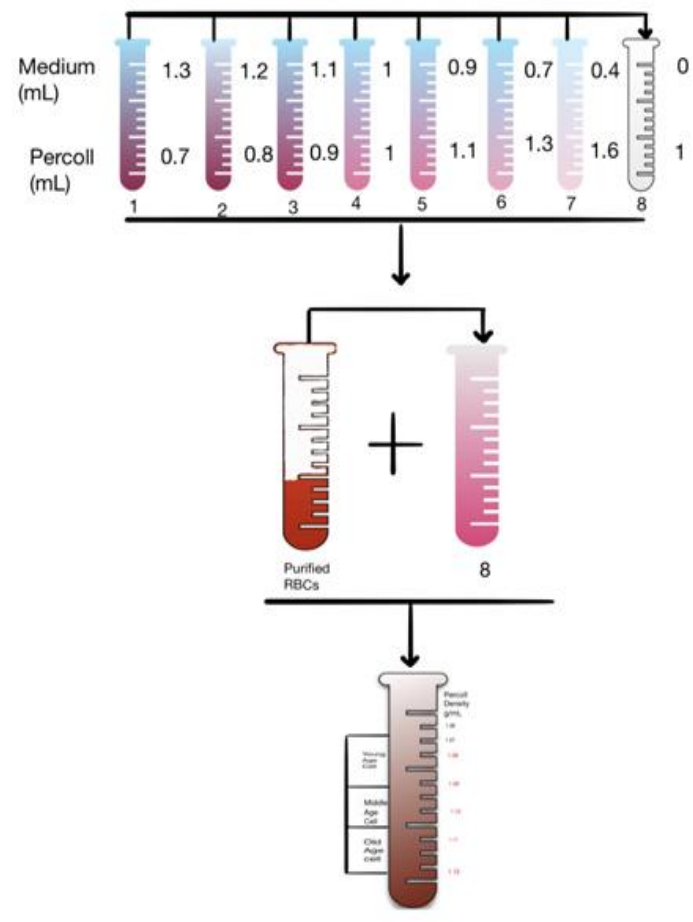

Fig1: Schematic representation of percoll density for sub-fractionated RBCs: $y=1.07-1.09, m=1.09$ $1.11, o=1.11-1.12 \mathrm{~g} / \mathrm{mL}$.

\subsection{RNA Extraction}

Total RNA was extracted from each age subfractionated RBCs of each sample using mirVanamiR Isolation kit (Invitrogen) according to the manufacturer's protocol. To 
Dysregulation of miR144 and miR451 Expression in the Circulating Human Erythrocytes from the African American Adults

begin, the age sub-fractioned RBC sample (400 $\mathrm{uL}$ ) disrupted in lysis solution. MiR homogenate additive was added to the sample, vortexed, and incubated for 10 minutes on ice. Acid-phenol chloroform was added in a volume equal to the total volume of the sample lysate, mixed, and centrifuged for 5 minutes at maximum speed at room temperature to extract. To extract, the upper phase removed carefully and transferred to a new tube. The RNA eluted in the elution solution. All samples stored at -80C.

\section{3. cDNA Synthesis and Mirna Quantification}

Real time PCR (RT-qPCR) was used for miR quantification in age sub-fractionated RBCs. Reverse transcription and PCR reaction were performed using TaqmanmiR transcription kit (Applied Biosystem). Applied biosystem uses total RNA that contains miR as the starting material for cDNA synthesis and allows for the detection of multiple miRs from a single cDNA preparation. The final reaction volume of reverse transcription was $20 \mathrm{uL}$. To quantify miR expression, a master mix was prepared following the manufacture's procedure (for each $10 \mathrm{uL}$ reaction: 7 uLTaqman master mix and 3 uL PCR primer). Amplification was carried out in 96 well plate using one step plus (Applied Biosystem) using the cycling conditions outlined by the manufacture. QRT-PCR was performed in duplication for each miRNA. RNU6 was used as a control.

\subsection{Data Analysis}

Differences in variance among the groups were analyzed using non-parametric one-way ANOVA. The differences in miRNAs expressions between control and T2DM were analyzed by using Student t-test. $\mathrm{P}<0.05$ was considered statistically significant. Data analysis was performed using stats tester software.

\section{RESULTS AND DISCUSSION}

\subsection{Differential Mir-144 and -451 Expression In Erythrocytes between Patients with T2DM and Control}

We selected two miRs to be measured in human erythrocyte obtained from control and T2DM AA. These miRs are highly expressed in erythrocytes and have a known protective function in these cells $[12,16]$. To identify if erythrocyte miRs expression differences in $\mathrm{y}, \mathrm{m}$, and $\mathrm{o}$ cells between control and T2DM individuals, we isolated total RNA from subfractionated purified erythrocytes and quantified miRs expression using qRT-PCR. MiR-451 was significantly reduced in T2DM group when compared to control group. Conversely, miR144 was significantly higher in T2DM when compared to control group (fig 2).

\section{Expression of miR-451 and miR-144}

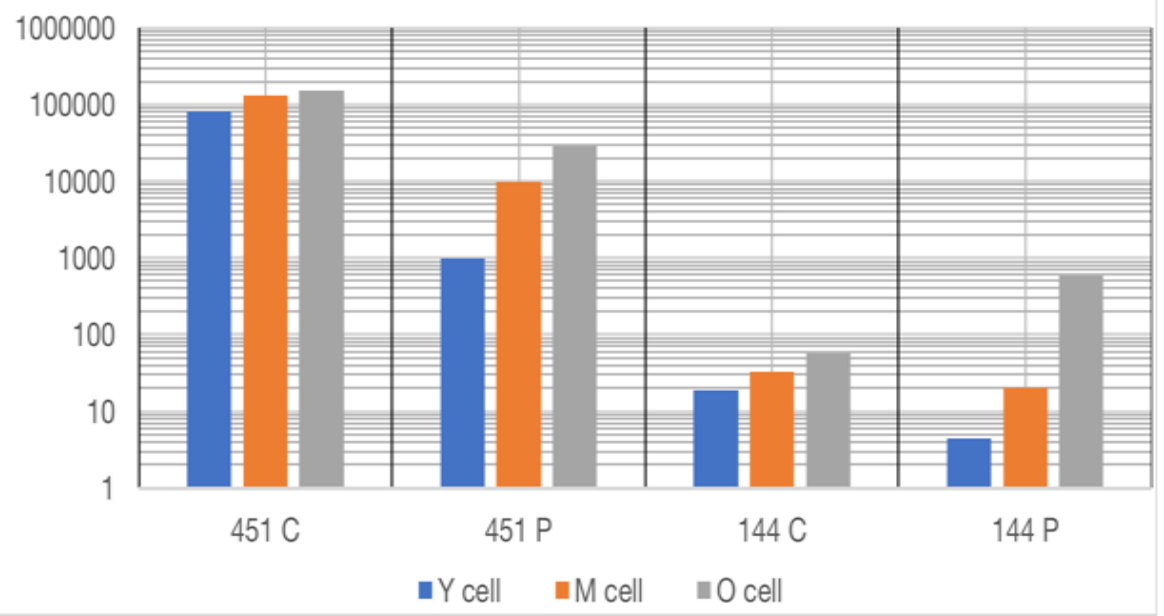

Fig2: Erythrocyte miR-451 and miR-144 expression in sub-fractionated erythrocytes in control and T2DM AA. MiR-451 expression control $(n=8)$ and T2DM ( $n=8)$. MiR-144 expression control $(n=8)$ and T2DM $(n=8)$. Expression was measured in duplicate. A non-parametric one-way ANOVA was used to determine statistical significance between both groups. $P<0.05$ considered significant.

We next sought to identify relationships between selected miRs expression in o cells and developing T2DM. We found there was a strong correlation between the miR-144 and development of T2DM. However, no such correlation with miR-451 was observed. The expression of miR-144 in o cells was significantly higher in T2DM group than control group (fig 3). 
Dysregulation of miR144 and miR451 Expression in the Circulating Human Erythrocytes from the African American Adults

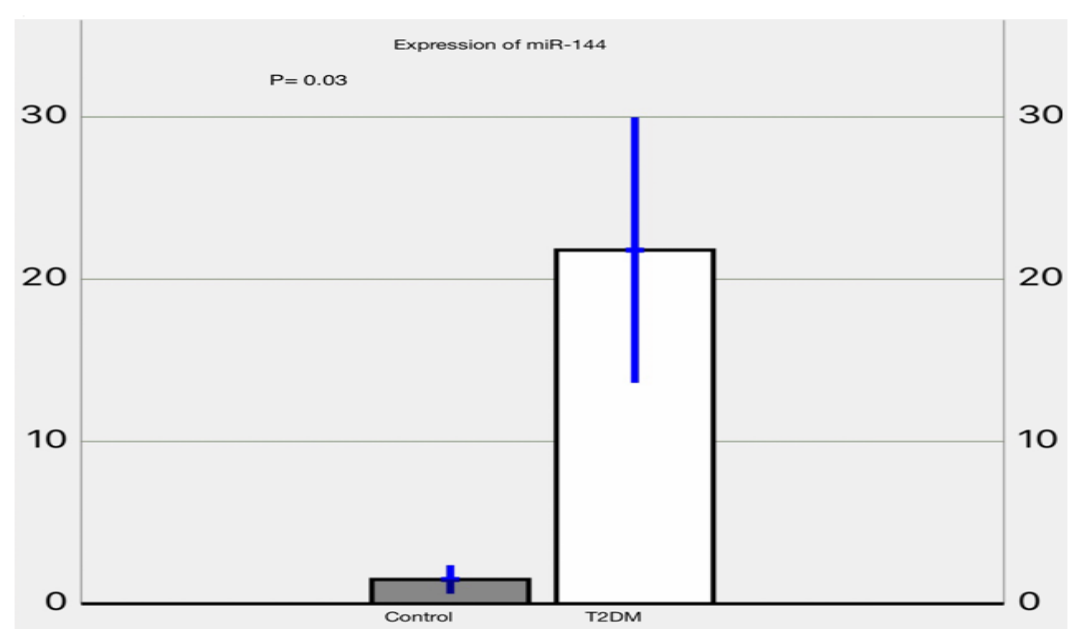

Fig3: Erythrocyte miR-144 expression in o cells in control and T2DM groups.

To determine the effect of T2DM on erythrocytes life span, we compared the expression of miR-451 in o cells between the two groups. We found a significant difference in miR-451 expression between control and
T2DM. As mentioned previously, miR-451 is responsible for reducing the oxidative stress in erythrocytes. We found that T2DM group has a significant decrease in miR-451 in comparison with control group (fig 4).

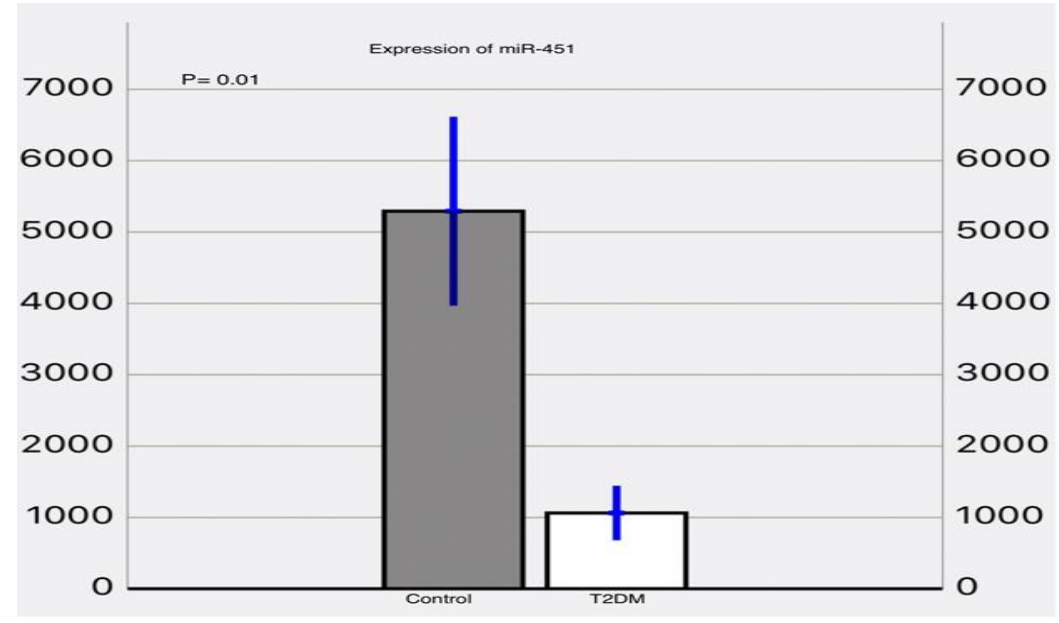

Fig4: Erythrocyte miR-451 expression in o cells in control and T2DM groups.

Many studies have been reported that circulatory miRs can be served as non-invasive biomarkers of diseases such as cancer, diabetes, and heart diseases. Few studies have explored miR expression in erythrocytes. This study explores the regulatory role of these miRs as potential indicators of cell aging. Our previous report showed the significant difference between miR-451 and miR-144 expression in subfractionated erythrocytes [17]. To our knowledge, this is the first study to investigate the expression of miRs in sub-fractionated erythrocytes ( $y, \mathrm{~m}$, and $\mathrm{o})$ of T2DM in comparison withthe controls. We reported that miR-451 are reduced in erythrocytes of the T2DM individuals. Also, miR-451 expressed more in o cells than $\mathrm{m}$ and $\mathrm{y}$ cells in both groups. MiR-451 regulates FOXO3 expression to protect erythrocytes from OS [18] by inhibiting the cytoplasmic adaptor protein 14-3$3 \zeta /$. This protein sequesters the production of FOXO3 that stimulates antioxidant genes such as catalase and glutathione peroxidases 1 [19].

Conversely, miR-144 expression was found to be up-regulated in T2DM in comparison with the control group. Moreover, miR-144 expressed more in o cells than $\mathrm{m}$ and y cells in both groups. MiR-144 expression correlate with several clinical characteristics associated with T2DM, including HbA1c making it an ideal candidate for genetic risk markers in AA. MiRNAs are reported to be involved in insulin production, secretion, and control insulin signaling pathway [20]. IRS proteins are known to be involved in the insulin signaling pathway. Dwi and colleagues found that miR-144 regulates IRS 1 gene [21]. Moreover, the study 
reported that there is a linear relationship between miR-144 and increasing glycemic status in T2DM. Another study showed that miR-144 knockout mice were insulin resistant. This finding proved that miR-144 plays an important role in insulin signaling pathway [21].

Both miR-451 and miR-144 are the most abundant miRs present in erythrocytes. The findings of our study are consistent with the other reports of reduced expression of miR-451 in individuals with certain condition such as diabetes lead to shortened life span of erythrocytes [22]. Also, miR-144 expression increased in T2DM group in comparison with control group. Our findings also suggest that $\mathrm{o}$ cells expressed more miR than $\mathrm{m}$ and y cells.

\section{Conclusion}

This is the first study that we are reporting an increase in the expression of miR-451 and miR144 in o cells than $\mathrm{m}$ and $\mathrm{y}$ cells in the erythrocytes from the individuals with T2DM. These findings could serve as potential biomarkers of red blood cell aging. These findings support similar cell aging studies. However, further studies are needed to clarify the potential role of RBC miRNAs as biomarkers for cell aging depending on the levels of which miR's that are altered during cell aging.

\section{ACKNOWLEDGMENT}

Authors thank fellows, clinicians and staff DTC for their kind support of this work.

Author's Contribution: "GA "GA and IJ conducted studies and drafted manuscript; RB, NH, GNB recruited patients; MBF edited manuscript; KKG designed and supervised experimental protocol."

\section{REFERENCES}

[1] International Diabetes Federation, 2019. IDF Atlas $9^{\text {th }}$ Edition. https://diabetesatlas.org/en/ resources/ Accessed January 10, 2020

[2] http://www.diabetes.org/diabetes-basics/ statistics/

[3] HaiyongChen, Hui-Yao Lan, Dimitrios $\mathrm{H}$ Roukos and William C Cho 2014. Application of microRNAs in diabetes mellitus. Body. http://joe.endocrinology-journals.org DOI: 10.1 530/JOE-13-0544.

[4] Huaije Bu, Sophia Wedel, Maria Cavinato, and Pidder Jansen-Dürr, 2017. MicroRNA Regulation of Oxidative Stress-Induced Cellular Senescence. Oxidative Medicine and Cellular Longevity. Volume 2017, Article ID 2398696, 12 pages https://doi.org/10. 1155/20 17/2398696.
[5] Pernow J, Mahdi A, Yang J, and Zhou Z, 2019. Red Blood Cell Dysfunction: a New Player in Cardiovascular Disease. Cardiovascular Research, 115, 1596-1605; doi:10.1093/cvr/ cvz156.

[6] Contreras-Zentella ML, Sanchez-Sevilla L, Suarez-Cuenca JA, Olguin-Martinez MG, Alatriste-Contreras M, Garcia-Garcia N, Orozco L, Hernandez-Munoz R, 2019. The role of oxidant stress and gender in the erythrocyte arginine metabolism and ammonia management in patients with type 2 diabetes. PLoS ONE 14(7): e0219481. https://doi.org/10.1371/ journal. pone. 0219481.

[7] Abdel-Moneim A, Abdel-Reheim ES, Semmler M, Addaleel W. The impact of glycemic status and metformin administration on red blood cell indices and oxidative stress in type 2 diabetic patients. Malays J Med Sci. 2019; 26(4):4760. https://doi.org/10.21315/mjms2019.26.4.6

[8] Xiaoqing Tang, Guiliang Tang, and SabireÖzcan 2008. Role of MicroRNAs in Diabetes. BiochimBiophys Acta. 2008 November; 1779(11): 697-701. Doi: 10.1016/j. bbagrm. 2008.06.010.

[9] Fluitt MB, Kumari N, Nunlee-Bland G, Nekahi S, Gambhir KK, 2016. MiRNA-15a, miRNA$15 \mathrm{~b}$, and miRNA-499 are reduced in Erythrocytes of Pre-Diabetic African-American Adults. Jacobs J Diabetes Endocrinol. 2016 Dec; 2(1): 014 PMID:29399662

[10] Fluitt MB, Kumari N, Nunlee-Bland G, Nekahi S, Gambhir KK, 2018. Energy Regulating Hormone Correlate with Erythrocyte MicroRNA Expression in Type 2 Diabetic African-American Adults. Jacobs J Diabetes Endocrinol. 2018 Dec; 3(3): 015

[11] Zampetaki A, Kiechl S, Drozdov I, Willeit P, Mayr U, Prokopi M, Mayr A, Weger S, Oberhollenzer F, Bonora E et al. 2010 Plasma microRNA profiling reveals loss of endothelial miR-126 and other microRNAs in type 2 diabetes. Circulation Research 107 810-817. (doi:10.1161/ CIRCRESAHA.110.226357)

[12] Yu D, dos Santos C, Zhao G, Jiang J, Amigo J, Khandros E, Dore L, Yao Y, D'Souza J, Zhang Z, Ghaffari S, Choi J, Friend S, Tong W, Orange J, Paw B, Weiss M, 2010. MiR-451 protects against erythroid oxidant stress by repressing 14-3-3z. Genes \& Development 24:1620-1633; doi:10.1101/gad.1942110

[13] Zhou S, Yang M, Zhao Y, Chen X, Zhang F, Li N, Yao P, Zhu T, Mei H, Wag S, Li Y, Chen S, Le Y, 2016. MicroRNA-451 Negatively Regulates Hepatic Glucose Production and Glucose Homeostasis by Targeting Glycerol Kinase-Mediated Gluconeogenesis. Diabetes, 65 (11), 3276-3288. 
[14] Zhang Z, Luo X, Ding S, Chen J, Chen T, Chen $\mathrm{X}$, Zha $\mathrm{H}$, Yao L, He $\mathrm{X}$ and Peng $\mathrm{H}$. MicroRNA-451 regulates p38 MAPK signaling by targeting of Ywhaz and suppresses the mesangial hypertrophy in early diabetic nephropathy. FEBS Lett. 2012;586:20-6.

[15] DwiSetyowati Karolina, Arunmozhiarasi Armugam, Subramaniam Tavintharan, Michael T. K. Wong, Su Chi Lim, Chee Fang Sum, KandiahJeyaseelan, 2011. MicroRNA 144 Impairs Insulin Signaling by Inhibiting the Expression of Insulin Receptor Substrate 1 in Type 2 Diabetes Mellitus.Plos One. 2011; 6(9): 10.1371.

[16] Rasmussen K, Simmini S, Abreu-Goodger C, Bartonicek N, Di Giacom M, Bilbao-Cortes D, Horos R, Von Lindern M, Enright A, O'Carroll $\mathrm{D}, 2010$. The miR-144/451 locus is required for erythroid homeostasis. J Exp Med Vol 207 No. 7 1351-1358; https://doi.org/10.1084/jem.2010 0458

[17] Jafri, I; Alsharif, G; Nunlee-Bland G, Gambhir, K.K., Erythrocyte miRNA 144 and miRNA 451 as Cell Aging Biomarkers in African American Adults, The Open Biochemistry Journal, DOI: 10.2174/1874091X01913010081, 2019, 13, 8187.

[18] Wanchen Wang, Chengwen Hang, Yanqing Zhang 1 ,Mingshi Chen 1, Xinyu Meng 1, Qing Cao 1, Nana Song 1, Jacobi Itkow 1, Feiyang Shen 1 and Duonan Yu, 2017. Dietary
miR-451 protects erythroid cells from oxidative stress via increasing the activity of Foxo3 pathway.Oncotarget. 2017; 8:107109-107124. https://doi.org/10.18632/oncotarget.22346.ww w.impactjournals.com

[19] Goud, B., Huet, C., and Louvard, D. (1985) J. Cell Biol. 100, 521-[15] Dragan Marinkovic, Xin Zhang, SafakYalcin, Julia P. Luciano, Carlo Brugnara, Tara Huber, and SaghiGhaffari, 2007. Foxo3 is required for the regulation of oxidative stress in erythropoiesis. The Journal of Clinical Investigation http://www.jci.org Volume 117 Number 8 August 2007.

[20] Juan Feng 1, Wanli Xing 1, 2, * and Lan Xie, 2016. Regulatory Roles of MicroRNAs in Diabetes. Int. J. Mol. Sci. 2016, 17, 1729; doi: 10.3390/ijms17101729.

[21] 21.DwiSetyowati Karolina, Arunmozhiarasi Armugam, Subramaniam Tavintharan, Michael T. K. Wong, Su Chi Lim, Chee Fang Sum, KandiahJeyaseelan, 2011. MicroRNA 144 Impairs Insulin Signaling by Inhibiting the Expression of Insulin Receptor Substrate 1 in Type 2 Diabetes Mellitus.

[22] Mazzanti L1, Faloia E, Rabini RA, Staffolani R, Kantar A, Fiorini R, Swoboda B, De Pirro R, Bertoli E, 1992. Diabetes mellitus induces red blood cell plasma membrane alterations possibly affecting the aging process. Clin Biochem. 1992 Feb; 25(1):41-6.

Citation: Ghadi Alsharif, Kanwal K Gambhir et. al. Dysregulation of miR144 and miR451 Expression in the Circulating Human Erythrocytes from the African American Adults. ARC Journal of Diabetes and Endocrinology. 2020; 6(1):1-6. doi:dx.doi.org/10.20431/2455-5983.0601001.

Copyright: (c) 2020 Authors. This is an open-access article distributed under the terms of the Creative Commons Attribution License, which permits unrestricted use, distribution, and reproduction in any medium, provided the original author and source are credited. 